\section{Endoscopic Management of Duodenal Diverticulitis Causing Common Bile Duct Obstruction and Cholangitis}

Second to the colon, the duodenum is the most common site for the development of diverticula in the alimentary tract. Inflammation of a juxtapapillary diverticulum is a rare complication, and may lead to compression of the common bile duct, resulting in cholangitis. We report here a case of this type, in which the patient was treated successfully using combined endoscopic therapy.

A 75-year-old woman presented with pain in the right upper abdomen. She had no history of cholecystitis or gallstones, but the further history included coronary heart disease, diabetes mellitus, reflux esophagitis, and immune thyroiditis. During continuing antibiotic treatment, the laboratory results showed elevated values for C-reactive protein $(320 \mathrm{mg} / \mathrm{l})$, bilirubin $(29 \mu \mathrm{mol} / \mathrm{l})$, and transaminases (alanine aminotransferase $113 \mathrm{U} / \mathrm{l}$ ). Due to cholestasis established by laboratory tests and confirmed by ultrasound, endoscopic retrograde cholangiopancreatography was carried out, and two duodenal diverticula were found.

The distal diverticulum showed no signs of inflammation, but a second diverticulum next to the papilla of Vater was hidden by the swollen mucosa. Irrigation of this diverticulum revealed a large amount of pus (Figure $\mathbf{1}$ ). Cannulation of the pa-

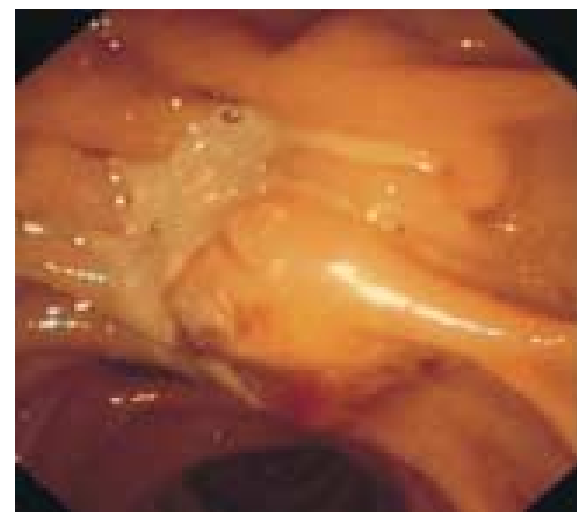

Figure 1 Endoscopic appearance of duodenal diverticulitis duct (b) pilla of Vater confirmed dilated bile ducts, with signs of cholangitis (secretion of bile and sludge). Sphincterotomy was carried out, and stenting of the common bile duct was performed (Figure 2). After this procedure, the patient's liver biochemistry returned to normal within a few days. A repeat ultrasound examination showed normal-caliber bile ducts, even after endoscopic removal of the stent.

The treatment of choice in patients suffering from duodenal diverticulitis and biliary obstruction remains unclear. A surgical approach can be used in patients with low surgical risk or with no surgical risk [1]. Due to the severe morbidity in the patient described here, an endoscopic approach was preferred in this case. Cholangitis was diagnosed after the diverticulum had been cleared, and it was therefore decided to promote the bile flow by stenting the common bile duct. In a similar case report, the authors did not cannulate the biliary system, in order to avoid ascending infection [2]. In contrast to the present case, the patient concerned was also suffering from cholestasis caused by a duodenal diverticulum, although no signs of cholangitis were described.

In conclusion, we believe that combined endoscopic therapy (irrigating the diverticulum and stenting the common bile duct) may offer an alternative to surgery in elderly patients with duodenal diverticulitis and secondary cholangitis.

\section{Caselitz, M. P. Manns, P. N. Meier}

Dept. of Gastroenterology

and Hepatology, Hannover Medical

School, Hannover, Germany

\section{References}

${ }^{1}$ San Roman AL, Moreira VF, Garcia M et al. Direct compression by a duodenal diverticulum causing common bile duct obstruction. Endoscopy 1994; 26: 334

2 Dickey W, Reilly M. Endoscopic management of food-filled duodenal diverticulum mimicking a pancreatic tumour. Endoscopy 1997; 29: S29-S30

\section{Corresponding Author}

\section{Caselitz, M.D.}

Dept. of Gastroenterology and Hepatology

Hannover Medical School

Carl-Neuberg-Strasse 1

30625 Hannover

Germany

Fax: $\quad+$ 49-511-5329405

E-mail: Caselitz.martin@ mh-hannover.de

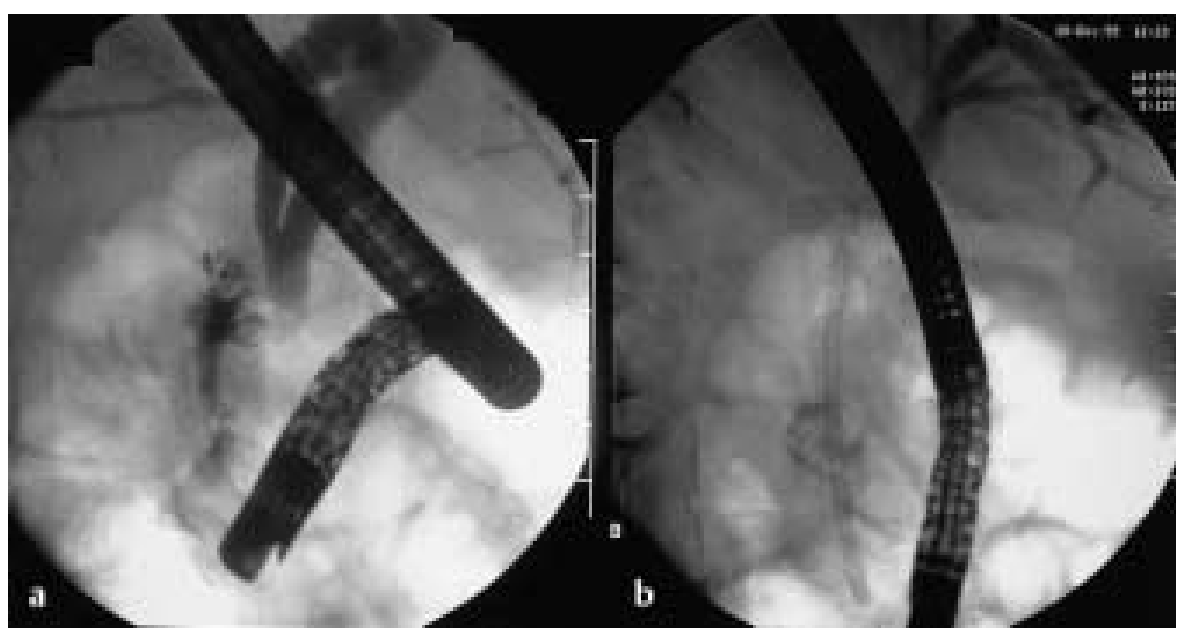

Figure 2 Radiography showing a duodenal diverticulum (a) and stenting of the common bile 